\title{
Interfactant action of an amphiphilic polymer upon directing graphene oxide layer formation on sapphire substrates
}

\author{
Yendry Regina Corrales Ureña', Welchy Leite Cavalcanti ${ }^{2}$, Marko Soltau ${ }^{3}$, Karolina Villalobos ${ }^{1}$, Klaus Rischka², \\ Paul-Ludwig Michael Noeske ${ }^{2^{*}}$, Kai Brune ${ }^{2}$ and Stefan Dieckhoff ${ }^{2}$
}

*Correspondence:

Michael.Noeske@ifam.

fraunhofer.de

${ }^{2}$ Adhesive Bonding

Technology and Surfaces,

Fraunhofer Institute

for Manufacturing

Technology and Advanced

Materials IFAM, Wiener Straße

12, 28359 Bremen, Germany

Full list of author information

is available at the end of the

article

\begin{abstract}
Quality assured surface pre-treatment may greatly enhance adhesive interactions and, thus, the performance and durability of material joints. This holds true as well for substrates used in coating processes as for adherents introduced into bonding processes. Wettable polymeric wetting agents_-shortly called polymeric interfactantscontribute to modifying surfaces and governing the properties of interphases. This is demonstrated for amphiphilic polymers directing the adsorption of graphene oxide (GO) nano-sheets from aqueous dispersion on alumina surfaces. In this contribution, contact angle measurements as well as X-ray photoelectron spectroscopy and scanning force microscopy investigations are applied for the characterization of thin films. $\mathrm{GO}$ is adsorbed either from a buffered dispersion on pristine aluminum oxide surfaces or on alumina modified with a few nanometers thin layer of a polymeric interfactant. Laterally extended nanoparticles and GO nano-sheets are preferentially found on interfactant layers whereas on pristine aluminum oxide smaller adsorbates dominate. The driving forces directing the $\mathrm{GO}$ attachment are discussed using a phenomenological model based on polymer/substrate interactions governing the sticking probabilities of GO nano-sheets with different sizes.
\end{abstract}

Keywords: Functional surface layers, Polymeric interfactants, Amhiphilic polymer, Graphene oxide immobilization, Aluminum oxide, Surface analysis

\section{Background}

Adhesive bonding technology and especially bonded light metal joints play an important role in vehicle and aircraft construction [1]. Among numerous approaches for the surface pretreatment of light metal adherents or substrates, layers formed by amphiphilic polymers may contribute in increasing the durability of adhesive joints or coatings [2,3]. The adsorption behavior of amphiphilic polymers on oxide, hydroxide or carbonate-based reaction layers on the surfaces of light metal alloys recently was investigated by dissipative particle dynamics (DPD) simulations $[4,5]$. The resulting some nanometers thin adsorbates were characterized by electron emission, e.g. using X-ray photoelectron spectroscopy (XPS) or optically stimulated electron emission (OSEE), by wetting techniques, and with respect to their chemical interaction with liquid water $[5,6]$. Concerning the film nucleation and growth of further moieties on top of the resulting polymer-coated surfaces, the

(c) The Author(s) 2017. This article is distributed under the terms of the Creative Commons Attribution 4.0 International License (http://creativecommons.org/licenses/by/4.0/), which permits unrestricted use, distribution, and reproduction in any medium, provided you give appropriate credit to the original author(s) and the source, provide a link to the Creative Commons license, and indicate if changes were made. 
feasible interface active agent (interfactant) effect with respect to directing the attachment of molecular films and the interaction with these films was discussed [6]. Based on this conception, polymeric interfactant films are two-dimensional layers with molecular dimensions, and they are made up of molecular entities in a way that each molecule spans between the solid substrate below it and the neighboring phase which extends over the half-space above it. Generally, this neighboring phase may be air, a liquid (like water [6]) or a solid, e.g. a cured adhesive or nano-particles. The interfactant layer may feature a homogeneous thickness all over the substrate, or it may exhibit some local variations in height leading to a difference between the roughness of the interfactant layer and the substrate roughness. A laterally homogeneous layer thickness is to be expected for layers of conventional adhesion promoters or coupling agents which exhibit an essentially linear head-tail structure, like surface-active material [4, 7-9]. The head group and often also the tail group are functional, and they may be different from each other. Depending on the length of the spacer (or backbone) unit between these terminal groups and on the temperature with respect to a critical temperature, the two-dimensional layers may be liquid, amorphous or highly ordered, e.g. forming a 2D crystalline layer in a self-assembly process resulting in a self-assembled monolayer (SAM) [8, 9]. Most pronounced during film growth, the molecular entities may be laterally mobile with respect to each otherwhich may hold true not only for the spacer chain and the tail group but also for the head group attached to the substrate. Such mobility of head groups is restricted in case of an intra-layer linking which is known for silane layers on oxide surfaces or also for alkyl thiol SAMs on gold surfaces showing disulphide links close to the substrate surface $[9,10]$. On the other hand, a laterally variable layer thickness may be expected for layers formed from polymers with a more complex shape than a linear one. As it was shown for amphiphilic polymers, such non-linear shape may result from intramolecular interactions, a phenomenon which is quite common also for polypeptides, e.g. globular proteins [4-6]. Especially for such polymeric monolayers the term polymeric interfactant layer was suggested recently [6].

The formation of graphene oxide (GO) layers on substrates is governed by the interactions between GO sheets (or flakes) and the substrate surface. GO may be considered a molecule with a hydrophobic polyaromatic backbone separated by cycloaliphatic structures containing $\mathrm{C}=\mathrm{C}$ double bonds and hydrophilic hydroxyl and epoxy groups; and the edges of this nearly flat carbon grid expose carboxylic acid groups $[11,12]$. The properties of reduced graphene oxide as a conducting material adsorbed on surfaces depend on the morphology of the constituting nano-sheets and the overall assembly arrangement [13]. Following the intended application of GO, reduced GO or graphene films, the morphology of the constituting adsorbed nano-sheets may be aspired to result smooth and flat or, alternatively, corrugated, e.g. wrinkled or crumpled [14-17]. The interaction between the substrate and graphene or between adsorbed nanoparticles and GO may govern the formation of wrinkles $[14,15]$ in the flexible nano-sheets. Wrinkles may occur on graphene planes and have high aspect ratios, with a height below $15 \mathrm{~nm}$ and lengths above $100 \mathrm{~nm}$ [15]. The layer assembly may be tuned by covalent interactions of the carboxylic and hydroxyl functional groups and by electrostatic interactions with polyelectrolytes $[18,19]$. Thin graphene oxide layers may be obtained by dipping hydrophilic substrates like pretreated quartz in a $70{ }^{\circ} \mathrm{C}$ hot aqueous dispersion of exfoliated 
GO, resulting in continuous and homogeneous films [20]. Chemical adsorption was investigated by $\mathrm{Ou}$ et al. who contacted an aqueous $\mathrm{GO}$ formulation for $12 \mathrm{~h}$ at $80^{\circ} \mathrm{C}$ with a (3-aminopropyl)triethoxysilane (APTES-SAM) covered Si wafer [21]. In a similar way, covalent anchoring was shown by $\mathrm{Su}$ and Chiou who attached GO to aminothiol modified gold surfaces [18]. Using such conventional adhesion promoter layers as substrates for the formation of GO films requires first the attachment of the organic layers to the respectively used substrate and, second, an adequate, e.g. chemical, interaction with the subsequently applied GO sheets. Therefore, polymeric interfactants may provide promising prospects since they strongly attach to a wide range of distinct substrate surfaces and, thus, may also be eligible for attaching GO nano-sheets.

In this contribution, we present insight into the adsorption of GO from aqueous dispersions on aluminum oxide surfaces modified with an interfactant layer based on a chemically non-reactive amphiphilic polymer. Our approach is highlighted in the sketch shown in Fig. 1 which is based on further developing the non-centrosymmetric layer conception recently described [6].

\section{Methods}

In this section, the experimental procedures applied for the manufacture of the layer systems on surfaces of aluminum oxide single crystals as well as the analysis methods used for characterizing the layers will be described.

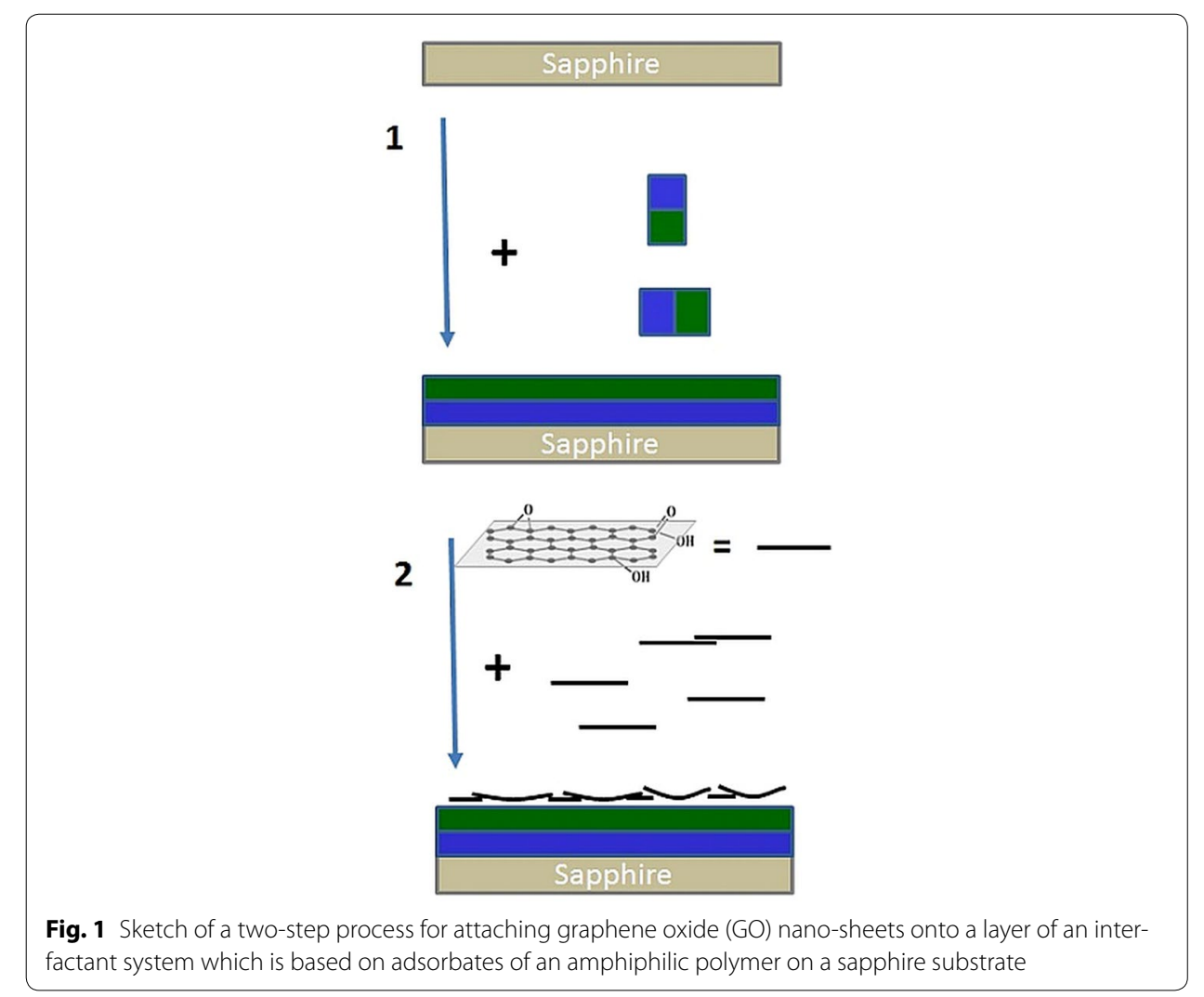




\section{Experimental procedures}

Sapphire ( $\alpha$-aluminum oxide, corundum single crystal) samples, single-side chemomechanically polished were purchased from TBL-Kelpin Dr. Gerd Lamprecht (Neuhausen, Germany).

A water-based formulation "G50 wb" containing the amphiphilic polymer Additive G50 (Straetmans High TAC GmbH, Hamburg, Germany) was used. The effective polymer will be denoted as "G50" throughout this contribution. Properties of the polymer formulation were described elsewhere $[5,6]$. Based on a parent formulation containing $4 \mathrm{wt} \%$ of organic constituents comprising polymer and triethanolamine (TEA) for adjusting the $\mathrm{pH}$ value, diluted formulations with a concentration of $2 \mathrm{wt} \%$ were prepared by adding demineralized water to the parent formulation.

A commercially available $4 \mathrm{mg} / \mathrm{mL}$ aqueous graphene oxide (GO) dispersion from Graphenea (San Sebastián, Spain) was used to prepare a formulation containing $0.5 \mathrm{mg} /$ $\mathrm{mL} \mathrm{GO}$ in $0.15 \mathrm{M}$ acetate buffer. Acetic acid and sodium acetate were used in reagent grade (Sigma-Aldrich).

The sapphire substrates were coated by immersion in the formulation based on Additive G50. Two distinct sequences of immersion and water rinsing were applied: $17 \mathrm{~h}$ of immersion and $45 \mathrm{~min}$ rinsing leading to samples labelled $\mathrm{G} 50-17 \mathrm{~h}$, and $27 \mathrm{~h}$ immersion and $4 \mathrm{~h}$ of rinsing providing samples labelled G50-27h. The GO dispersion was homogenized by applying an ultrasonic treatment for $5 \mathrm{~min}$ before use. After $1 \mathrm{~h}$ in contact with the GO dispersion, the coated substrates were rinsed gently for some seconds with water and then submerged in deionized water for $2 \mathrm{~min}$; followed by blowing with air. Finally, the samples were allowed to dry and were stored under environmental conditions at room temperature.

\section{Analysis methods}

Investigations of the surface composition were performed with X-ray photoelectron spectroscopy (XPS). XPS spectra with an information depth of around $0.01 \mu \mathrm{m}$ were taken using a Kratos Ultra system applying excitation of photoelectrons by monochromatic $\mathrm{Al} \mathrm{K}_{\alpha}$ radiation within an area of approximately $0.2 \mathrm{~mm}^{2}$. The system was operated at a base pressure of $4 \times 10^{-8} \mathrm{~Pa}$, the sample neutralization was performed with low energy electrons $(<5 \mathrm{eV})$. An electrostatic lens was used, the take-off angle of electrons was $0^{\circ}$, and the pass energy was fixed to $20 \mathrm{eV}$ (or, respectively, $40 \mathrm{eV}$ in case of some less concentrated constituents) in high resolution spectra and $160 \mathrm{eV}$ in survey spectra. Elemental ratios were calculated based on the area of the peaks and considering relative sensitivity factors. Atomic concentrations for the detected elements are given in atomic percent, abbreviated by at\%. The given at $\%$ values may be translated to weight percent values by considering the respective atomic masses. For each sample two positions were investigated. When calculating the thickness of adsorbates, a compact and homogeneous layer is assumed. The equation of $d=-\ln (y) \times X$ was employed, in which $d$ is the thickness of the layers, $y$ is the Al2p signal intensity ratio between covered and pristine $\mathrm{Al}_{2} \mathrm{O}_{3}$ samples, and $\mathrm{X}$ is the inelastic mean free path of Al2p electrons, assumed to be $3.3 \mathrm{~nm}$ in an organic adsorbate layer [22].

The sample surface topography was analyzed using scanning force microscopy (SFM). Two distinct instrumental setups were applied. An SFM from Asylum Research was 
operated in the tapping mode in air. Silicon probes (model Tap150Al-G, back side of the cantilever covered with $\mathrm{Al}$ ) with a resonance frequency of $150 \mathrm{kHz}$ and a force constant of $5 \mathrm{~N} / \mathrm{m}$ were used. A scanning probe microscope operated in the SFM 'tapping mode' in air (Digital Instruments Nanoscope III multimode with phase extender box) was employed profiting from a maximum scan range of the scanner around $100 \mu \mathrm{m}$. Si cantilevers (Nanosensors) with a resonance frequency around $250 \mathrm{kHz}$ corresponding to force constants around $20 \mathrm{~N} / \mathrm{m}$ were used. The nominal tip diameter was in the range of $10 \mathrm{~nm}$. For characterizing the structures of the adsorbates, height differences, among other criteria, were evaluated. The values reported are an average of at least ten height differences measured.

The apparent contact angles were measured using a goniometer (ramé-hart instrument co., USA) by sessile drop technique, and MiliQ grade water was used as probe liquid; the volume of the drops was constant $(10 \mu \mathrm{L})$ for each measurement at a temperature of $22{ }^{\circ} \mathrm{C}$. The contact angle values reported are an average value of at least three separate drops on different substrate areas. The recorded images were analyzed by Drop Image ramé-hart instruments software.

\section{Results and discussion}

In this section, we will highlight and discuss characteristics of adsorbing graphene oxide from a buffered aqueous dispersion on pristine aluminum oxide surfaces and on $\mathrm{Al}_{2} \mathrm{O}_{3}$ surfaces covered with thin films of an amphiphilic polymer denoted as G50.

Initially, the layer formation and stability of G50 layers on sapphire surfaces was studied spectroscopically using XPS. The respective $\mathrm{Al}_{2} \mathrm{O}_{3}$ substrate was submerged for a period of 17 or $27 \mathrm{~h}$ in the aqueous formulation and subsequently rinsed for $45 \mathrm{~min}$ or $4 \mathrm{~h}$, respectively. Table 1 and Fig. 2 indicate the obtained elemental composition of the films based on the XPS results. After G50 adsorption the intensity of the Al2p and F1s photoelectron emission from the substrate is roughly halved. Within the films carbon, oxygen and nitrogen species are detected, with similar concentrations $[\mathrm{C}],[\mathrm{O}]$ and $[\mathrm{N}]$ when comparing G50-17h and G50-27h samples. Stable layers withstanding several hours of rinsing with water were formed. An adsorbate thickness of $3.1 \pm 0.2 \mathrm{~nm}$ is obtained for G50-17h and a marginally lower thickness for G50-27h.

The findings of contact angle measurements are presented with microscopic images in Fig. 3, and the evaluation of the investigations is given in Table 2. After depositing the

Table 1 Results of XPS investigations, with surface concentrations given in atomic \% (at\%), for distinct pristine sapphire samples (average values are given) and for these substrates after contact with 2 wt\% G50 formulation either for $17 \mathrm{~h}$ and then rinsed $45 \mathrm{~min}$ (samples G50-17h) or for $27 \mathrm{~h}$ and then rinsed $4 \mathrm{~h}$ (samples G50-27h)

\begin{tabular}{|c|c|c|c|c|c|}
\hline \multirow[t]{2}{*}{ Sample } & \multicolumn{5}{|l|}{ Element } \\
\hline & $\mathrm{F}$ & 0 & C & $\mathbf{N}$ & Al \\
\hline Pristine sapphire & $1.0 \pm 0.1$ & $43.7 \pm 0.5$ & $20.3 \pm 0.7$ & 0.1 & $34.0 \pm 0.5$ \\
\hline G50-17h, sample 1 & $0.4 \pm 0.1$ & $28.6 \pm 2.2$ & $52.1 \pm 5.1$ & $0.5 \pm 0.1$ & $16.1 \pm 3$ \\
\hline G50-17h, sample 2 & $0.3 \pm 0.1$ & $27.0 \pm 0.7$ & $55.9 \pm 2.3$ & $0.5 \pm 0.1$ & $16 \pm 1.6$ \\
\hline G50-27h, sample 1 & $0.4 \pm 0.1$ & $32.8 \pm 1.7$ & $47.6 \pm 3.0$ & $0.4 \pm 0.1$ & $22.7 \pm 1.4$ \\
\hline G50-27h, sample 2 & $0.3 \pm 0.1$ & $29.3 \pm 0.7$ & $51.0 \pm 1.6$ & $0.4 \pm 0.1$ & $18.9 \pm 0.9$ \\
\hline
\end{tabular}




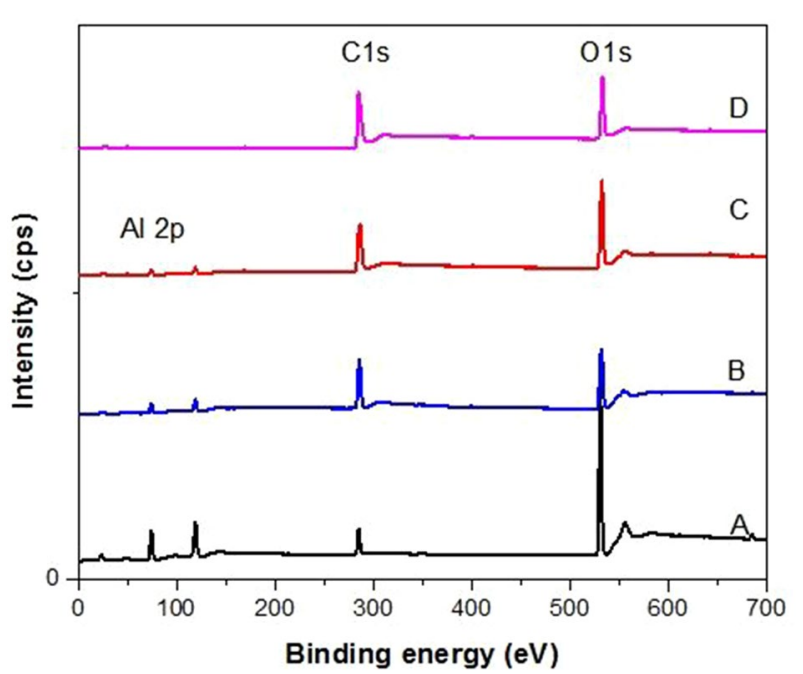

Fig. 2 XPS results obtained in the binding energy region between 0 and $700 \mathrm{eV}$ for $A$ a pristine sapphire surface, $B$ a G50/sapphire interfactant film (sample G50-17h), C a GO/G50/sapphire film, and D a dried deposit of water-rinsed $\mathrm{GO}$ dispersion

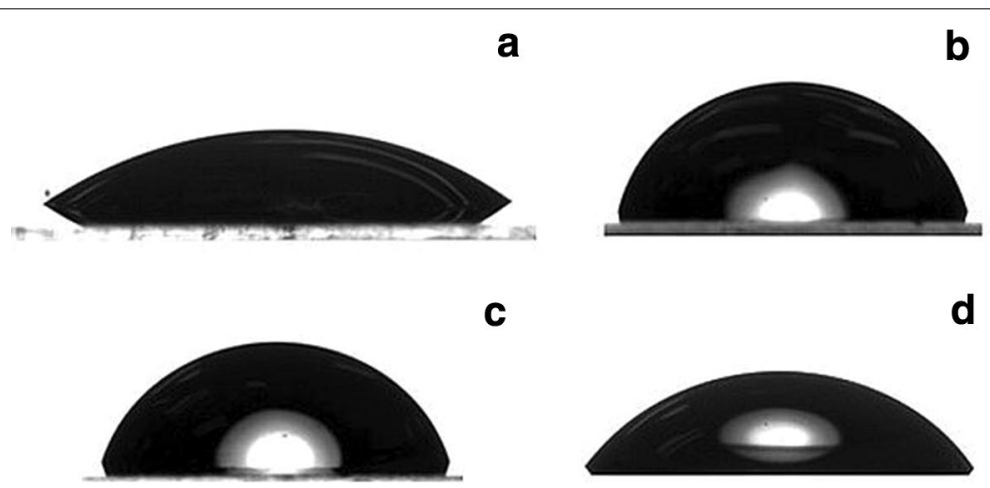

Fig. 3 Light microscopic image of a water drop on a a dried deposit of water-rinsed GO dispersion, $\mathbf{b}$ a pristine sapphire surface, c a G50/sapphire interfactant film (sample G50-17h), and d a GO/G50/sapphire film

Table 2 Results of contact angle investigations with the contact angles given in ${ }^{\circ}$, as performed for pristine sapphire, a G50/sapphire film (sample G50-17h), a GO/sapphire adsorbate, a GO/G50/sapphire film, and a dried deposit of GO dispersion (intensively rinsed with water)

\begin{tabular}{lc}
\hline Sample & As-deposited \\
\hline Pristine sapphire & $76.9 \pm 1.1$ \\
G50/sapphire & $72.2 \pm 3$ \\
GO/sapphire & $65.5 \pm 4$ \\
GO/G50/sapphire & $53.1 \pm 2.1$ \\
Dried GO dispersion & $36 \pm 2$ \\
\hline
\end{tabular}

G50 layer on top of the sapphire substrate, the contact angle changes from $76.9 \pm 1.1^{\circ}$ to $72.2 \pm 3^{\circ}$. Depending on the surface pre-treatment and the resulting coverage with carbonaceous species, water contact angle values in the range between $<5^{\circ}$ and $72^{\circ}$ 
were reported for alumina substrates [23, 24]. Therefore, the rather high contact angle measured is attributed to surface species which increase the hydrophobicity, e.g. carbonaceous moieties with a concentration around 20 at \% as indicated by the XPS measurements. The contact angle found for the G50-17 h sample results similar to the one reported by Gonçalves et al. [5] for a G50 film formed on a magnesium alloy surface.

The microscopic findings for the surface structure of pristine sapphire surfaces and the G50-17h sample as obtained by SFM investigations are presented in Fig. 4a on a larger scale and in Fig. $5 \mathrm{a}$ and $\mathrm{b}$ on a smaller scale. For the pristine sapphire surface a rather smooth topography is obtained, characterized by an $R_{a}$ roughness value of $0.9 \mathrm{~nm}$. This finding is interpreted to result from the chemo-mechanical surface treatment of the sapphire sample. In contrast, for annealed crystals a step-terrace surface morphology was found [25]. The deposition of the G50 film leads to a decrease in surface roughness, and an $R_{a}$ value of $0.3 \mathrm{~nm}$ is obtained.

The solid constituents of the GO dispersion were characterized assessing a dried deposit of the dispersion which subsequently was rinsed with water. Water rinsing reduced the concentration of $\mathrm{Mn}, \mathrm{N}$ and S species in the topmost surface of the bulky dried deposit. Finally, minor residues of the underlying moieties with concentrations below 1 at\% were obtained, as highlighted in Table 3 . This finding is attributed to a layerwise build-up of the deposit which results from the drying process characterized by the loss of the aqueous dispersant or solvent, respectively. During the advance of drying, presumably the agglomeration of graphene oxide sheets precedes the deposition of more soluble species which accordingly may be dissolved by rinsing with water after the

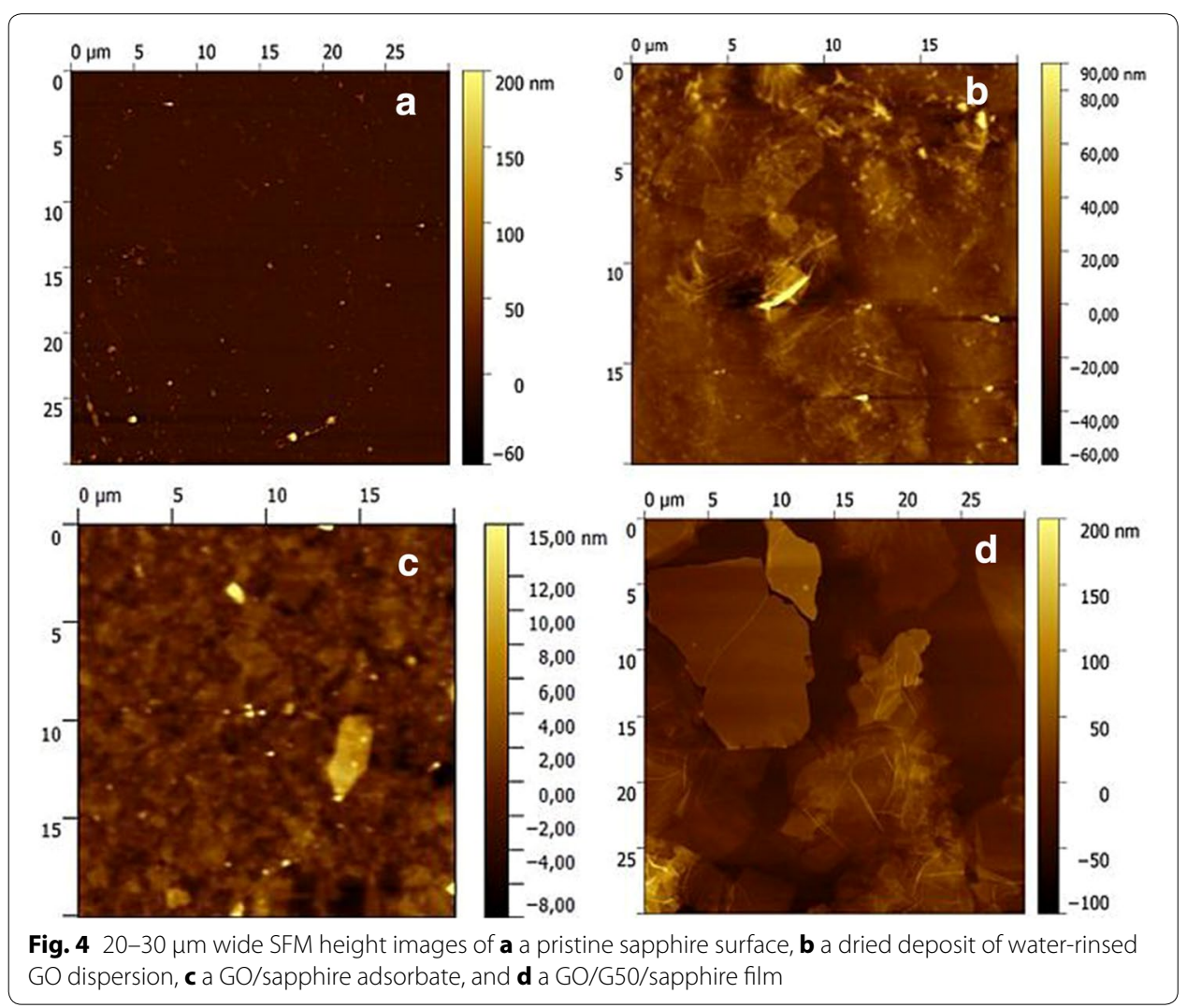




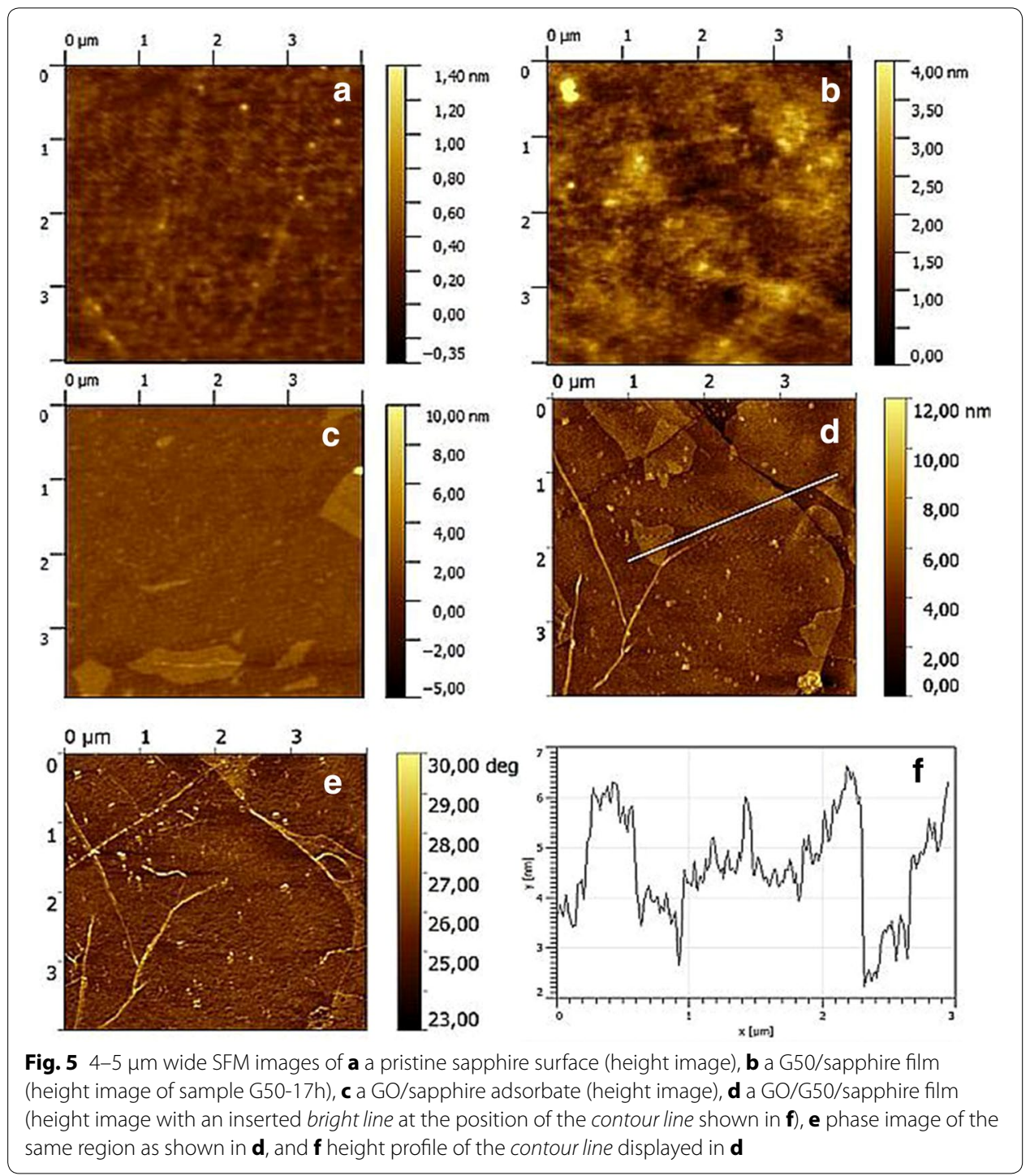

Table 3 Results of XPS investigations, with surface concentrations given in atomic \% (at\%), performed for a dried deposit of GO dispersion (intensively rinsed with water), pristine sapphire, a G50/sapphire film (sample G50-17h), and a GO/G50/sapphire film

\begin{tabular}{|c|c|c|c|c|c|c|c|}
\hline \multirow[t]{2}{*}{ Sample } & \multicolumn{7}{|l|}{ Element } \\
\hline & $F$ & Mn & 0 & $S$ & C & $\mathrm{N}$ & Al \\
\hline Dried GO dispersion & - & $0.2 \pm 0.3$ & $22.5 \pm 0.4$ & $0.1 \pm 0.1$ & $76.5 \pm 0.5$ & $0.5 \pm 0.1$ & - \\
\hline Pristine sapphire & $1.0 \pm 0.1$ & - & $44.2 \pm 0.1$ & - & $20.5 \pm 0.4$ & $0.2 \pm 0.1$ & $33.9 \pm 0.1$ \\
\hline G50/sapphire & $0.3 \pm 0.1$ & - & $27.0 \pm 0.7$ & & $55.9 \pm 2.3$ & $0.5 \pm 0.1$ & $16.0 \pm 1.6$ \\
\hline GO/G50/sapphire & - & $0.3 \pm 0.1$ & $30.0 \pm 0.4$ & $0.6 \pm 0.1$ & $61.6 \pm 0.9$ & $0.6 \pm 0.1$ & $7.3 \pm 1.7$ \\
\hline
\end{tabular}

drying. The wetting behavior of the resulting solid was characterized by a water contact angle around $36^{\circ}$ (cf. Table 2). This is in agreement with findings by Dai et al. [26] who reported a water contact angle of $34^{\circ}$ for GO. Additionally, the presence of molecular species with S- and N-containing species as found by XPS may influence the contact angle [27]. As shown in Fig. 4b, SFM investigations reveal distinct morphological 
features: on the one hand more than $10 \mu \mathrm{m}$ wide regions with wrinkled sheets oriented parallel to the surface, and on the other hand regions with considerably smaller deposits.

The deposition of graphene oxide on sapphire surfaces modified with G50 was performed from buffered GO dispersions during an immersion period of $1 \mathrm{~h}$. The respective survey scans obtained from XPS investigations are shown in Fig. 2 in the spectra labelled $\mathrm{B}$ and $\mathrm{C}$. As highlighted in Table 3, the Al2p signal intensity decreased as compared to the sample G50-17h due to material deposition from the GO dispersion. In detail, besides $\mathrm{C}$ - and $\mathrm{O}$-containing species also $\mathrm{Mn}$ and $\mathrm{S}$ species and probably $\mathrm{N}$-containing species were deposited. In addition, XPS signals with a high spectral resolution in the C1s region (not shown) reveal similar spectroscopic features for the interfactant G50 and the graphene oxide. Two dominant C1s peaks centered around 285 and $287 \mathrm{eV}$ are attributed to hydrocarbonaceous species with $\mathrm{C}^{*}-\mathrm{H}$ or $\mathrm{C}^{*}-\mathrm{C}$ bonds and, respectively, to species with $\mathrm{C}^{*-}-\mathrm{O}$ single bonds. On a molecular level, these ones are assigned to polyoxyalkylene moieties in G50-based polymers and to functional groups in graphene oxide, like carbon-bonded hydroxyls, phenols, ethers, or epoxy moieties [5, 28]. As a consequence, the adsorption of moieties from the GO dispersion will be inferred from evaluating the attenuation of the sapphire-related Al2p XPS signal intensity, from contact angle measurements, and from SFM investigations. Subsequently, the thus obtained findings will be described.

Based on evaluating the Al2p peak intensities listed in Table 3, the adsorbate layer found for the GO/G50/sapphire sample is $2.2 \pm 0.5 \mathrm{~nm}$ thicker than the one observed for the G50-17h sample. This finding is ascribed to the formation of a GO-based adsorbate layer with an effective average thickness of $2.2 \mathrm{~nm}$ on top the G50 adlayer. Accordingly, as shown in Table 2 the water contact angle decreases from $72.2 \pm 3^{\circ}$ to $53.1 \pm 2.1^{\circ}$ which is attributed to the formation of a more hydrophilic surface termination. However, the wettability of the deposit formed from a dried GO dispersion and characterized by a contact angle of $36 \pm 2^{\circ}$ is not reached. This finding may be reflected on the base of the SFM images obtained for the GO/G50/sapphire sample as displayed in Figs. 4d, $5 \mathrm{~d}$ and e, as well as in Fig. 6a and c. The microscopic investigations indicate that after contact with the GO dispersion the smooth G50 adlayer is largely-but not completelycovered by particulate adsorbates of different sizes and morphologies. Thus, the wettability of the sample may be affected by both the regions covered by G50 and the ones covered by GO particles; and the resulting contact angle of $53^{\circ}$ is in between $72^{\circ}$ and $36^{\circ}$. Considering the SFM images in more detail, the $30 \mu \mathrm{m}$ wide height image in Fig. $4 \mathrm{~d}$

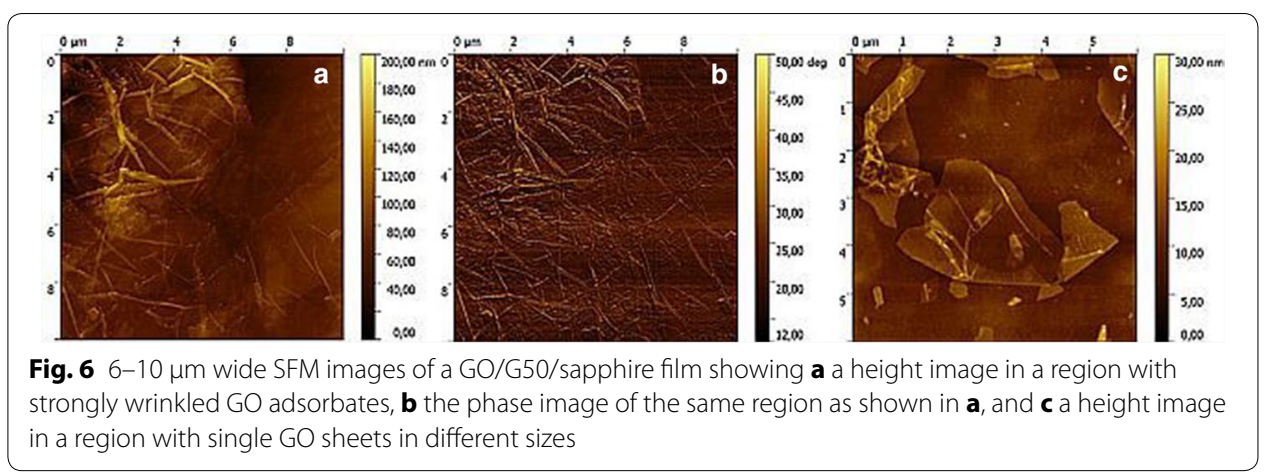


comprised sections of around ten particles with the particle width ranging between 5 and $15 \mu \mathrm{m}$. Noticeably, the particle widths were more than one order of magnitude larger than the particle heights which indicates that the largest face of the particles preferentially was oriented parallel to the surface of the G50 adlayer. The actually exposed particle faces show significantly different surface morphologies, ranging from smooth to rough and strongly wrinkled areas. The SFM height image in Fig. 6a and the phase image in Fig. 6b acquired in the same region revealed that bunches of corrugated nanosheets with wrinkle heights of several ten nanometers may extend over at least $10 \mu \mathrm{m}$ wide regions. In contrast to such strongly corrugated multilayer regions, the SFM height images in Figs. 5d and 6c show adsorbates made up of smooth and several micrometers wide graphene oxide sheets with heights between 1 and $5 \mathrm{~nm}$. The wrinkle density was characterized by around five wrinkles per $10 \mu \mathrm{m}^{2}$ area, and the wrinkles were a few micrometers long and some nanometers high. For comparison, single layer graphene flakes decorated with oxygen- and hydrogen-containing groups typically show an average height in the range of 0.8 to $1.2 \mathrm{~nm}$ [29]. On top of the sheet presented in Fig. 5d, ad-islands with smaller sheets in parallel orientation are indicated in the height image. As these islands are not contrasted in the correspondent phase image shown in Fig. 5e, they were attributed to a material with a similar deformation behavior as the GO sheet, and they are interpreted to originate from GO alike. Moreover, the phase image clearly highlights that the topographically lower region in the gap towards the nearby GO sheet at the right edge of the images in Fig. $5 \mathrm{~d}$ and e were composed of a material with a different deformation behavior as compared to GO. This material is interpreted to be the polymeric interfactant layer covering the alumina surface. The height line in the cross section shown in Fig. $5 \mathrm{f}$ highlights the heights of the sheets and, as well, the considerable corrugation of the sheet surface. For the nano-sheet shown in Fig. 6c intramolecular folding was observed since the sheet edge pointing towards the bottom of the image shows a significantly greater height difference with respect to the underlying G50 layer, and it appeared back-folded with an elevated outward tab region. In contrast to the region shown in Fig. 6a, the regions imaged in Figs. 5d and 6c exhibited relatively low GO coverages, and overlapping between neighboring smooth GO sheets was not a dominant feature.

Finally, pristine sapphire substrates were immersed in buffered aqueous GO dispersion, and a change of surface properties was observed. The water contact angle decreased from $76.9 \pm 1.1^{\circ}$ to $65.5 \pm 4^{\circ}$, and the surface roughness $R_{a}$ inferred from SFM images increased from 0.9 to $1.8 \pm 0.7 \mathrm{~nm}$. For a more detailed discussion, SFM height images are shown in Fig. 4c and on a smaller scale in Fig. 5c. The surface of the GO/sapphire sample was clearly more corrugated than the surface of pristine sapphire. When comparing the surface structure of GO/sapphire adsorbates with the one of GO/G50/ sapphire films, it became clear that in contrast to the latter one the $\mathrm{GO} /$ sapphire sample did not manifest particulate adsorbates with a particle width above $5 \mu \mathrm{m}$, and especially extended GO nano-sheets were not imaged when investigating more than ten surface regions.

These findings indicate significant effects of the G50 interfactant layer with respect to directing the adsorption of graphene oxide nano-sheets towards a face-to-substrate adsorption geometry, favoring adsorbates from huge GO sheets with the cyclic ether 
groups on the sheet surface oriented towards the linear aliphatic ether groups in the adsorbate layer formed by the amphiphilic polymer G50. Aiming at substantiating the inferred interaction between GO sheets and the G50 interfactant layers, both the GO/ sapphire sample and the GO/G50/sapphire sample were subjected to prolonged rinsing with water. Concerning the GO/G50/sapphire films, longer-term rinsing resulted in decreasing the concentration of $\mathrm{Mn}$ - or S-containing species by approximately $50 \%$ as compared to the XPS findings reported in Table 3 for the shortly rinsed GO/G50/ sapphire films. The Al2p signal intensity was not significantly affected by such rinsing which indicates that the film thickness did not change substantially. After immersion in water under gentle stirring for one hour the samples were imaged again by SFM, and the obtained SFM images are shown in Fig. 7.

Concerning the GO/G50/sapphire films, the SFM height images in Fig. 7c and d show regions with adjacent single GO sheets extended horizontally over the surface. This finding indicated some robustness of the nano-sheet adsorbates against rinsing with water. Concerning GO/sapphire adsorbates, in Fig. $7 a$ a region with an $R_{a}$ roughness value of $3.1 \pm 0.5 \mathrm{~nm}$ is presented which is higher than the value of $1.8 \pm 0.7 \mathrm{~nm}$ obtained before rinsing. The SFM phase image in Fig. 7b was acquired in the same region; and the phase contrast indicates three types of domains with distinct deformability. The domains with the lowest phase angle coincided with topographically low areas and, therefore, were attributed to the sapphire substrate. In contrast, the domains with the highest phase angle corresponded with topographically high areas which were around $6 \mathrm{~nm}$ higher than the substrate and dominantly were laterally extended by several $100 \mathrm{~nm}$.

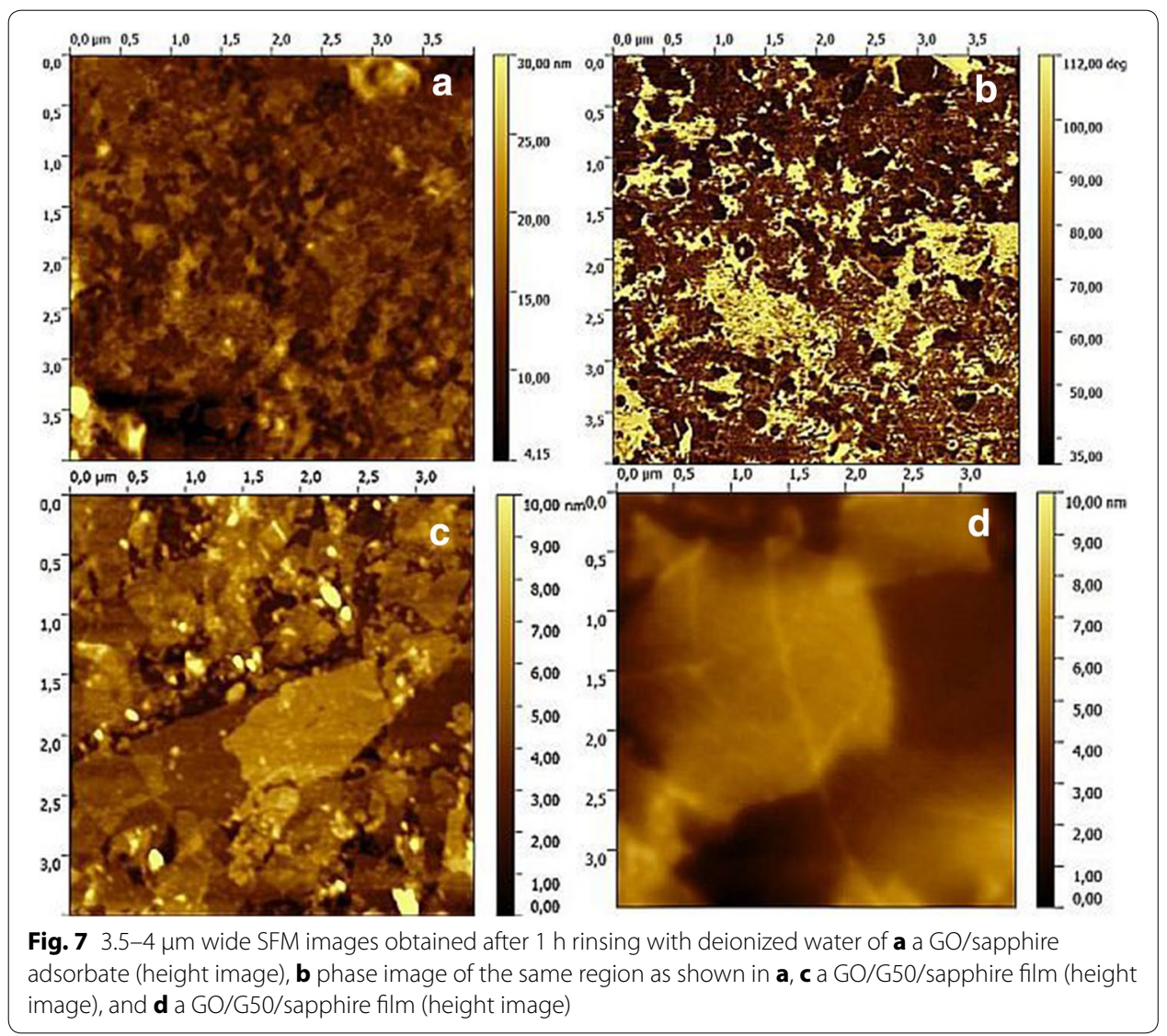


These domains were interpreted as elevated graphene oxide adsorbates. However, they appeared rougher and more pitted than the GO sheets shown in Fig. $7 \mathrm{c}$ and d. Also, the material constituting the third domain appeared significantly rough and scattered; and straight edges of adsorbates or the voids in between them were hardly discerned.

Summarizing, the adsorbates found on $\mathrm{GO} /$ sapphire samples appeared to expose a much longer contour length of their lateral boundaries as compared to the much wider particulate adsorbates which were characteristic for GO/G50/sapphire films. Somehow, the interfactant layers seemed to hinder the random deposition of GO-based material and to favor the attachment of several micrometer wide graphene oxide sheets. A driving force for the latter aspect was discussed above based on dominating contributions from van der Waals interactions. A possible driving force for the deposition of smaller particles in case of the $\mathrm{GO} /$ sapphire system is highlighted subsequently, based on polar and electrostatic contributions. The $\mathrm{pH}$ value of the buffer solution was 4.75 , GO suspensions are characterized by a negative $\zeta$-potential at $\mathrm{pH}$ values around 5 , and alumina and probably also sapphire surfaces are characterized by positive $\zeta$-potentials at these $\mathrm{pH}$ values [30-32]. Therefore, favorable polar and electrostatic interactions may be expected between GO and sapphire surfaces. Predominantly the edges of GO sheets are regions which are rich in acidic groups [14,33]. Therefore, the adsorption of GO sheets with a high ratio of functionalized edges may be preferred on sapphire as compared to the adsorption of GO sheets with epoxy-functionalized faces. Demonstratively, this promoting ratio will be the higher the smaller GO sheets are, and the competing effect will be lower for smaller GO sheets. In this way, the finding of smaller particulate adsorbates in case of the GO/sapphire system than in case of the GO/G50/sapphire system may be expectable. Still, possible influences of water-soluble, S- and N-containing molecular species in the GO dispersion will need to be assessed to embrace all the interactions competing with the interfactant action of the amphiphilic polymer layer.

\section{Conclusions}

An amphiphilic polymer was used as interface active agent (interfactant) governing interphase properties during the adsorption of graphene oxide (GO) particles. The interfactant directed the adsorption of graphene oxide (GO) nano-sheets from aqueous dispersion on sapphire surfaces in a face-to-substrate geometry, favoring the adsorption of several micrometers wide sheets to the adsorption of smaller and more hydrophilic entities. The study provides a model system illustrating how assembly of nano-particles or the attachment of polymers could be controlled due to interfactant nano-layers formed on various oxide surfaces.

\section{Authors' contributions}

YC and KR developed and worked out the protocol for graphene oxide immobilization. MS developed, performed and — with MN — adapted the formulation of the amphiphilic polymer used as an interfactant. WLC and MN adapted the methodology of interfactant layer formation for sapphire substrates. YC and MN performed and evaluated Scanning Probe Microscopy and XPS investigations. KV and YC performed and evaluated contact angle measurements. YC, WLC, KR and $\mathrm{MN}$ took part in setting up the experiments and in analysing and merging the obtained data. WLC, KR, MN, KB and SD contributed in the conceptual approach and in discussing the obtained data. YC and MN drafted the manuscript. All authors read and approved the final manuscript.

\section{Author details}

${ }^{1}$ National Laboratory of Nanotechnology LANOTEC, $1.3 \mathrm{~km}$ North from the USA Embassy, San José, Costa Rica. ${ }^{2}$ Adhesive Bonding Technology and Surfaces, Fraunhofer Institute for Manufacturing Technology and Advanced Materials IFAM, Wiener Straße 12, 28359 Bremen, Germany. ${ }^{3}$ Straetmans High TAC GmbH, Merkurring 94, 22143 Hamburg, Germany. 


\section{Acknowledgements}

The authors are grateful to Dr. Hauke Brüning for fruitful discussions.

\section{Competing interests}

The authors declare that they have no competing interests.

\section{Availability of data and materials}

All relevant data of the article are presented in the manuscript (in tables or figures).

\section{Publisher's Note}

Springer Nature remains neutral with regard to jurisdictional claims in published maps and institutional affiliations.

Received: 5 January 2017 Accepted: 4 May 2017

Published online: 15 May 2017

\section{References}

1. Brockmann W, Hennemann OD, Kollek H, Matz C. Adhesion in bonded aluminium joints for aircraft construction. Int J Adhes Adhes. 1986;6:115-43. doi:10.1016/0143-7496(86)90016-3.

2. Cavalcanti WL, Brinkmann A, Noeske M, Buchbach S, Straetmans F, Soltau M. Anticorrosive systems-dual-purpose defenders. Eur Coat J. 2012;10:30-3.

3. Scharf S, Noeske M, Cavalcanti WL, Schiffels P. Multi-functional, self-healing coatings for corrosion protection: materials, design and processing. In: Makhlouf AH, editor. Handbook of smart coatings for materials protection. Cambridge: Woodhead Publishing; 2014. doi:10.1533/9780857096883.1.75.

4. Cavalcanti WL, Noeske PLM. Investigating dynamic interactions by multi-scale modelling: from theory to applications. Chem Model. 2014;11:175-200. doi:10.1039/9781782620112-00175.

5. Gonçalves LMG, Sanchez LC, Stamboroski S, Urena YRC, Cavalcanti WL, Ihde J, Noeske M, Soltau M, Brune K. Instantly investigating the adsorption of polymeric corrosion inhibitors on magnesium alloys by surface analysis under ambient conditions. J Surf Eng Mater Adv Technol. 2014;4:282-94. doi:10.4236/jsemat.2014.45032.

6. Stamboroski S, Stachera PN, Ureña YRC, Hrycyna GH, Ribas Neto WIT, de Azambuja WK, Salz D, Ihde J, Noeske PLM, Cavalcanti WL. Implementation of diverse non-centrosymmetric layer concepts for tuning the interface activity of a magnesium alloy. Appl Adhes Sci. 2016;4:6. doi:10.1186/s40563-016-0063-7.

7. Schreiber F. Structure and growth of self-assembling monolayers. Prog Surf Sci. 2000;65:151-256. doi:10.1016/ S0079-6816(00)00024-1.

8. Onclin S, Ravoo BJ, Reinhoudt DN. Engineering silicon oxide surfaces using self-assembled monolayers. Angew Chem Int Ed. 2005;44:6282-304. doi:10.1002/anie.200500633.

9. Ulman A. Formation and Structure of self-assembled monolayers. Chem Rev. 1996;96:1533-54. doi:10.1021/cr9502357.

10. Fenter P, Eberhardt A, Eisenberger P. Self-assembly of n-alkyl thiols as disulfides on Au(111). Science. 1994;266:12168. doi:10.1126/science.266.5188.1216.

11. He H, Klinowski J, Forster M, Lerf A. A new structural model for graphite oxide. Chem Phys Lett. 1998;287:53-6. doi:10.1016/S0009-2614(98)00144-4.

12. Zhang Y, Wu C, Guo S, Zhang J. Interactions of graphene and graphene oxide with proteins and peptides. Nanotechnol Rev. 2013;2:27-45. doi:10.1515/ntrev-2012-0078.

13. Palma CA, Samorì P. Blueprinting macromolecular electronics. Nat Chem. 2011;3:431-6. doi:10.1038/nchem.1043.

14. Wang F, Liu J. Evaporation induced wrinkling of graphene oxide at the nanoparticle interface. Nanoscale. 2015;7:919-23. doi:10.1039/c4nr05832a.

15. Deng S, Berry V. Wrinkled, rippled and crumpled graphene: an overview of formation mechanism, electronic properties, and applications. Mater Today. 2016;19:197-212. doi:10.1016/j.mattod.2015.10.002

16. Chen PY, Sodhi J, Qiu Y, Valentin TM, Steinberg RS, Wang Z, Hurt RH, Wong IY. Multiscale graphene topographies programmed by sequential mechanical deformation. Adv Mater. 2016:28:3564-71. doi:10.1002/adma.201506194.

17. Zou F, Zhou H, Jeong DY, Kwon J, Eom SU, Park TJ, Hong SW, Lee J. Wrinkled surface-mediated antibacterial activity of graphene oxide nanosheets. ACS Appl Mater Interfaces. 2017:9:1343-51. doi:10.1021/acsami.6b15085.

18. Su PG, Chiou CF. Electrical and humidity-sensing properties of reduced graphene oxide thin film fabricated by layer-bylayer with covalent anchoring on flexible substrate. Sensors Actuators B. 2014;200:9-18. doi:10.1016/j.snb.2014.04.035.

19. Zhang D, Tong J, Xia B, Xue Q. Ultrahigh performance humidity sensor based on layer-by-layer self-assembly of graphene oxide/polyelectrolyte nanocomposite film. Sensors Actuators B. 2014;203:263-70. doi:10.1016/j. snb.2014.06.116.

20. Wang X, Zhi L, Müllen K. Transparent, conductive graphene electrodes for dye-sensitized solar cells. Nano Lett. 2008:8:323-7. doi:10.1021/nl072838r.

21. Ou J, Wang J, Liu S, Mu B, Ren J, Wang H, Yang S. Tribology study of reduced graphene oxide sheets on silicon substrate synthesized via covalent assembly. Langmuir. 2010;26:15830-6. doi:10.1021/la102862d.

22. Esplandiu MJ, Noeske PLM. XPS investigations on the interactions of 1,6-hexanedithiol/Au(111) layers with metallic and ionic silver species. Appl Surf Sci. 2002;199:166-82. doi:10.1016/S0169-4332(02)00608-6.

23. Zhang D, Wang Y, Gan Y. Characterization of critically cleaned sapphire single-crystal substrates by atomic force microscopy, XPS and contact angle measurements. Appl Surf Sci. 2013;274:405-17. doi:10.1016/j. apsusc.2012.12.143.

24. González-Martín ML, Labajos-Broncano L, Jańczuk B, Bruque JM. Wettability and surface free energy of zirconia ceramics and their constituents. J Mater Sci. 1999:34:5923-6. doi:10.1023/A:1004767914895. 
25. Witek G, Noeske M, Mestl G, Shaikhutdinov SK, Behm RJ. Interaction of platinum colloids with single crystalline oxide and graphite substrates: a combined AFM, STM and XPS study. Catal Lett. 1996;37:35-9. doi:10.1007/BF00813516.

26. Dai J, Wang G, Ma L. Study on the surface energies and dispersibility of graphene oxide and its derivatives. J Mater Sci. 2015;50:3895-907. doi:10.1007/s10853-015-8934-z.

27. Wang S, Zhang Y, Abidi N, Cabrales L. Wettability and surface free energy of graphene films. Langmuir. 2009;25:11078-81. doi:10.1021/la901402f.

28. Larciprete R, Fabris S, Sun T, Lacovig P, Baraldi A, Lizzit S. Dual path mechanism in the thermal reduction of graphene oxide. J Am Chem Soc. 2011;133:17315-21. doi:10.1021/ja205168x.

29. Spyrou K, Rudolf P. An introduction to graphene. In: Georgakilas V, editor. Functionalization of graphene. New York: Wiley; 2014. doi:10.1002/9783527672790.ch1.

30. Corrales Ureña YR, Wittig L, Vieira Nascimento M, Faccioni JL, Noronha Lisboa Filho P, Rischka K. Influences of the $\mathrm{pH}$ on the adsorption properties of an antimicrobial peptide on titanium surfaces. Appl Adhes Sci. 2015;3:7. doi:10.1186/s40563-015-0032-6.

31. Lei Z, Christov N, Zhao XS. Intercalation of mesoporous carbon spheres between reduced graphene oxide sheets for preparing high-rate supercapacitor electrodes. Energy Environ Sci. 2011;4:1866-73. doi:10.1039/c1ee01094h.

32. Franks GV, Meagher L. The isoelectric points of sapphire crystals and alpha-alumina powder. Colloids Surf A: Physicochem Eng Asp. 2003;214:99-110. doi:10.1016/S0927-7757(02)00366-7.

33. Shen X, Lin X, Yousefi N, Jia J, Kim JK. Wrinkling in graphene sheets and graphene oxide papers. Carbon. 2014;66:8492. doi:10.1016/j.carbon.2013.08.046

\section{Submit your manuscript to a SpringerOpen ${ }^{\circ}$ journal and benefit from:}

- Convenient online submission

\section{- Rigorous peer review}

- Immediate publication on acceptance

- Open access: articles freely available online

- High visibility within the field

- Retaining the copyright to your article

Submit your next manuscript at $\boldsymbol{\nabla}$ springeropen.com 